\title{
Correction to: The Dynamic Fault Tree Rare Event Simulator
}

Carlos E. Budde (iD), Enno Ruijters (D), and Mariëlle Stoelinga (iD)

\section{Correction to:}

Chapter "The Dynamic Fault Tree Rare Event Simulator" in: M. Gribaudo et al. (Eds.): Quantitative Evaluation

of Systems, LNCS 12289,

https://doi.org/10.1007/978-3-030-59854-9_17

In the original version of this chapter Reference 5 was published incorrectly. Reference 5 has now been corrected. 